\title{
A lab in the field: High-frequency analysis of water quality and stable isotopes in streamwater and precipitation
}

Jana von Freyberg ${ }^{1,2}$, Bjørn Studer ${ }^{1}$, James W. Kirchner ${ }^{1,2}$

${ }^{1}$ Department of Environmental Systems Science, ETH Zurich, Zurich, Switzerland

$5 \quad{ }^{2}$ Swiss Federal Research Institute WSL, Birmensdorf, Switzerland

Correspondence to: Jana von Freyberg (jana.vonfreyberg@usys.ethz.ch) 


\section{Supplement}

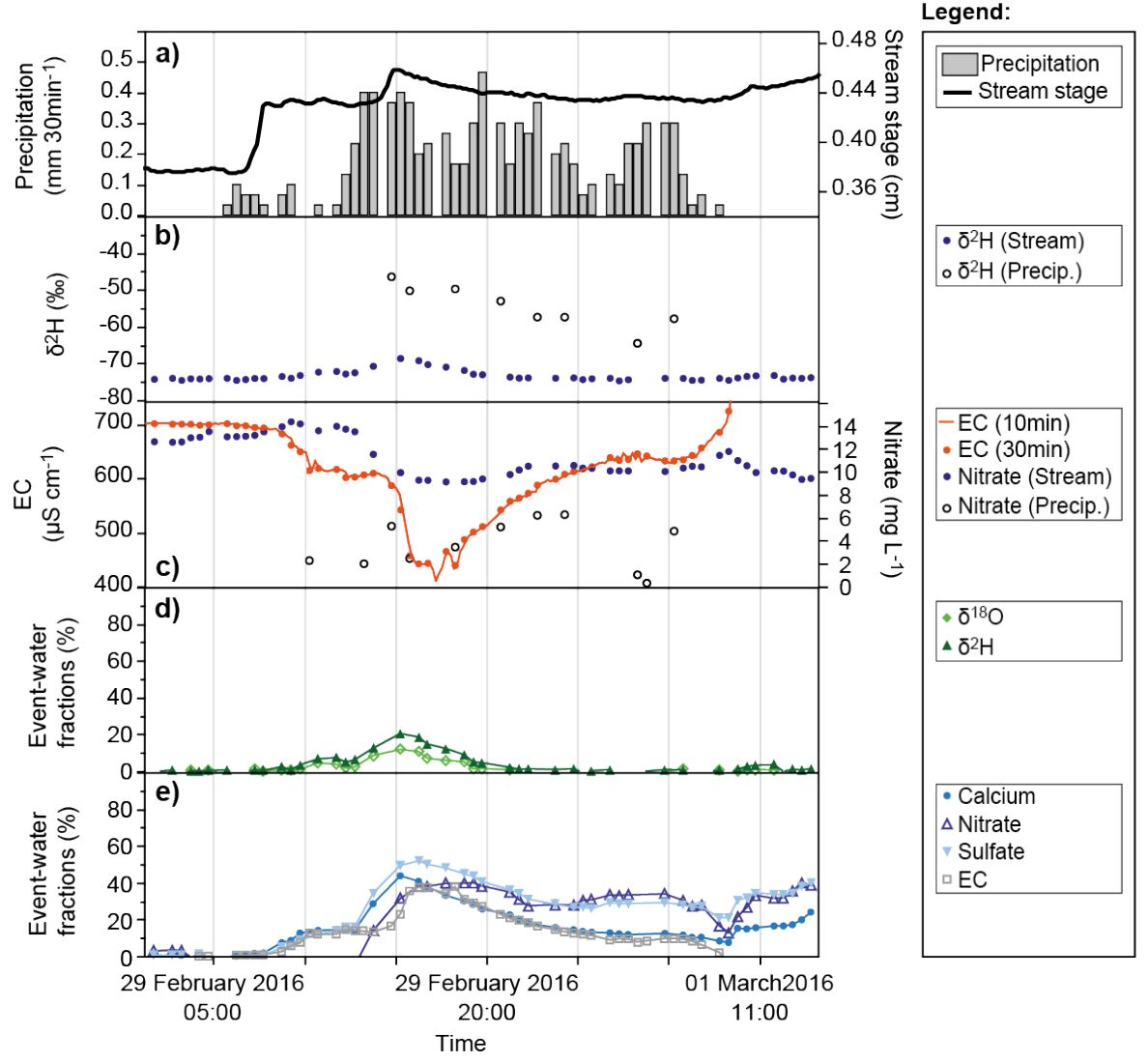

Figure S1: Precipitation Event \#5 together with the hydrologic (a), isotopic (b) and chemical (c) responses in streamwater. Panels d) and e) show the fractions of event-water based on isotopic hydrograph separation (IHS) and chemical hydrograph separation 5 (CHS), respectively, which are different for both types of tracers: While the IHS yields event-water fractions smaller than $20 \%$, CHS based on chemical tracers estimated much larger event-water fractions of more than $40 \%$. 
Table S 1: End-members and event-water fractions during peak flow.

\begin{tabular}{|c|c|c|c|c|c|c|}
\hline & $\delta^{18} \mathrm{O}(\%)$ & $\delta^{2} H(\%)$ & $\mathrm{Ca}^{2+}\left(\mathrm{mg} \mathrm{L}^{-1}\right)$ & $\mathrm{NO}_{3}^{-}\left(\mathrm{mg} \mathrm{L}^{-1}\right)$ & $\mathrm{SO}_{4}^{2-}\left(\mathrm{mg} \mathrm{L}^{-1}\right)$ & $\mathrm{EC}\left(\mu \mathrm{S} \mathrm{cm}^{-1}\right)$ \\
\hline Event & \multicolumn{6}{|c|}{ Pre-event-water end member $\left(C_{\mathrm{P}}\right) \pm S E_{\mathrm{CP}}$} \\
\hline$\# 1$ & $-10.98 \pm 0.03$ & $-75.10 \pm 0.20$ & $162.84 \pm 1.84$ & $11.33 \pm 0.05$ & $20.22 \pm 1.27$ & $708.80 \pm 18.45$ \\
\hline$\# 2$ & $-10.61 \pm 0.07$ & $-73.36 \pm 0.29$ & $166.66 \pm 1.58$ & $13.85 \pm 0.12$ & $24.18 \pm 1.49$ & $718.40 \pm 14.39$ \\
\hline \#3 & $-10.71 \pm 0.03$ & $-73.57 \pm 0.18$ & $166.28 \pm 1.62$ & $12.84 \pm 0.10$ & $23.77 \pm 1.47$ & $711.20 \pm 14.28$ \\
\hline \#4 & $-10.77 \pm 0.05$ & $-73.74 \pm 0.32$ & $158.11 \pm 1.53$ & $11.18 \pm 0.08$ & $21.84 \pm 1.35$ & $667.00 \pm 13.88$ \\
\hline$\# \mathbf{5}$ & $-10.78 \pm 0.03$ & $-74.24 \pm 0.20$ & $163.97 \pm 1.66$ & $12.94 \pm 0.18$ & $24.46 \pm 1.51$ & $700.40 \pm 14.01$ \\
\hline \#6 & $-11.25 \pm 0.03$ & $-77.30 \pm 0.21$ & $137.90 \pm 1.86$ & $8.05 \pm 0.10$ & $14.23 \pm 0.92$ & $562.20 \pm 12.61$ \\
\hline \#7 & $-10.93 \pm 0.03$ & $-75.52 \pm 0.18$ & $163.61 \pm 1.57$ & $11.52 \pm 0.12$ & $21.05 \pm 1.30$ & $694.40 \pm 13.93$ \\
\hline$\# 8$ & $-11.06 \pm 0.04$ & $-76.20 \pm 0.21$ & $166.67 \pm 1.61$ & $11.66 \pm 0.10$ & $22.22 \pm 1.37$ & $696.80 \pm 14.09$ \\
\hline Event & \multicolumn{6}{|c|}{ Event-water end member $\left(C_{\mathrm{E}}\right) \pm S E_{\mathrm{CE}}$ at peak flow } \\
\hline \#1 & $-13.00 \pm 0.26$ & $-91.86 \pm 3.65$ & $13.14 \pm 3.19$ & $0.38 \pm 0.22$ & $0.12 \pm 0.06$ & $53.88 \pm 24.97$ \\
\hline$\# 2$ & $-5.62 \pm 0.30$ & $-55.71 \pm 2.57$ & $15.90 \pm 2.37$ & $1.63 \pm 0.66$ & $1.27 \pm 0.63$ & $15.80 \pm 20.69$ \\
\hline \#3 & $-8.42 \pm 0.73$ & $-61.52 \pm 4.44$ & $16.31 \pm 0.89$ & $0.46 \pm 0.13$ & $0.07 \pm 0.04$ & $24.02 \pm 20.34$ \\
\hline$\# 4$ & $-10.70 \pm 0.54$ & $-94.29 \pm 4.36$ & $10.05 \pm 1.69$ & $1.84 \pm 0.30$ & $0.05 \pm 0.04$ & $5.91 \pm 20.07$ \\
\hline$\# \mathbf{5}$ & $-5.92 \pm 0.09$ & $-46.96 \pm 1.32$ & $13.76 \pm 1.01$ & $3.39 \pm 0.71$ & $2.39 \pm 0.60$ & $12.87 \pm 20.07$ \\
\hline$\# 6$ & $-11.87 \pm 0.40$ & $-86.22 \pm 2.21$ & $6.99 \pm 3.76$ & $0.31 \pm 0.16$ & $0.05 \pm 0.04$ & $10.58 \pm 20.92$ \\
\hline$\# 7$ & $-14.58 \pm 1.06$ & $-130.11 \pm 6.16$ & $10.81 \pm 2.21$ & $1.28 \pm 0.50$ & $0.06 \pm 0.04$ & $18.15 \pm 21.15$ \\
\hline$\# 8$ & $-15.29 \pm 0.50$ & $-121.41 \pm 2.76$ & $12.15 \pm 2.56$ & $2.72 \pm 0.45$ & $0.17 \pm 0.15$ & $20.24 \pm 20.98$ \\
\hline Event & \multicolumn{6}{|c|}{ Streamwater end member $\left(C_{\mathrm{S}}\right) \pm S E_{\mathrm{CS}}$ at peak flow } \\
\hline$\# 1$ & $-11.85 \pm 0.03$ & $-82.19 \pm 0.17$ & $78.00 \pm 0.78$ & $4.16 \pm 0.03$ & $7.12 \pm 0.46$ & $414.00 \pm 8.28$ \\
\hline$\# 2$ & $-10.27 \pm 0.03$ & $-70.60 \pm 0.17$ & $64.60 \pm 0.66$ & $3.73 \pm 0.03$ & $5.12 \pm 0.34$ & $264.00 \pm 5.28$ \\
\hline \#3 & $-10.04 \pm 0.03$ & $-69.28 \pm 0.17$ & $89.92 \pm 0.89$ & $6.26 \pm 0.04$ & $10.52 \pm 0.67$ & $346.00 \pm 6.92$ \\
\hline \#4 & $-12.54 \pm 0.03$ & $-87.74 \pm 0.17$ & $90.82 \pm 0.90$ & $5.39 \pm 0.04$ & $8.00 \pm 0.52$ & $428.00 \pm 8.56$ \\
\hline \#5 & $-10.20 \pm 0.03$ & $-68.66 \pm 0.17$ & $98.23 \pm 0.96$ & $9.94 \pm 0.04$ & $13.56 \pm 0.85$ & $542.00 \pm 10.84$ \\
\hline \#6 & $-11.58 \pm 0.03$ & $-80.06 \pm 0.17$ & $107.28 \pm 1.04$ & $5.48 \pm 0.04$ & $9.57 \pm 0.61$ & $336.00 \pm 7.24$ \\
\hline$\# 7$ & $-15.12 \pm 0.03$ & $-106.78 \pm 0.17$ & $91.31 \pm 0.90$ & $4.58 \pm 0.04$ & $7.59 \pm 0.49$ & $366.00 \pm 7.32$ \\
\hline$\# 8$ & $-12.54 \pm 0.03$ & $-85.85 \pm 0.17$ & $142.95 \pm 1.36$ & $9.65 \pm 0.04$ & $16.71 \pm 1.04$ & $1338.00 \pm 26.76$ \\
\hline
\end{tabular}

\begin{tabular}{|c|c|c|c|c|c|c|}
\hline \multirow[b]{2}{*}{ Event } & \multicolumn{6}{|c|}{ Event-water fraction $F_{\mathrm{E}} \pm S E(\%)$ at peak flow } \\
\hline & $\delta^{18} \mathbf{O}$ & $\delta^{2} \mathbf{H}$ & $\mathrm{Ca}^{2+}$ & $\mathrm{NO}_{3}^{-}$ & $\mathrm{SO}_{4}{ }^{2-}$ & EC \\
\hline$\# 1$ & $42.85 \pm 5.80$ & $42.29 \pm 9.28$ & $56.67 \pm 1.42$ & $65.45 \pm 1.35$ & $65.21 \pm 3.20$ & $45.01 \pm 2.64$ \\
\hline$\# 2$ & $6.72 \pm 1.49$ & $15.68 \pm 2.84$ & $67.70 \pm 1.20$ & $82.84 \pm 4.50$ & $83.17 \pm 2.95$ & $64.67 \pm 2.17$ \\
\hline$\# 3$ & $29.21 \pm 9.43$ & $35.61 \pm 13.23$ & $50.92 \pm 0.85$ & $53.15 \pm 0.75$ & $55.93 \pm 3.92$ & $53.15 \pm 2.11$ \\
\hline \#4 & $-{ }^{a}$ & $68.15 \pm 14.48$ & $45.45 \pm 0.98$ & $61.99 \pm 2.06$ & $63.53 \pm 3.28$ & $36.15 \pm 2.16$ \\
\hline \#5 & $11.79 \pm 0.85$ & $20.44 \pm 1.30$ & $43.77 \pm 0.94$ & $31.43 \pm 2.69$ & $49.40 \pm 5.35$ & $23.04 \pm 2.32$ \\
\hline \#6 & $52.06 \pm 34.19$ & $30.90 \pm 8.05$ & $23.39 \pm 1.51$ & $33.24 \pm 1.24$ & $32.92 \pm 6.13$ & $36.29 \pm 2.40$ \\
\hline \#7 & $114.79 \pm 33.35$ & $57.27 \pm 6.47$ & $47.32 \pm 1.05$ & $67.77 \pm 3.34$ & $64.11 \pm 3.24$ & $48.56 \pm 2.15$ \\
\hline$\# \mathbf{8}$ & $35.03 \pm 4.22$ & $21.36 \pm 1.40$ & $15.35 \pm 1.27$ & $22.44 \pm 1.49$ & $25.02 \pm 6.63$ & $-94.77 \pm 6.38^{b}$ \\
\hline
\end{tabular}

${ }^{\mathrm{a}}$ Unrealistic event-water fractions were obtained because the $\delta^{18} \mathrm{O}$ signatures in precipitation and streamwater were too similar.

${ }^{\mathrm{b}}$ Wash-off of road salt resulted in unrealistic event-water fractions based on EC. 
Table S 2: End-members and event-water fractions during maximum event-water fraction.

\begin{tabular}{|c|c|c|c|c|c|c|}
\hline & $\delta^{18} \mathrm{O}(\%)$ & $\delta^{2} H(\%)$ & $\mathrm{Ca}^{2+}\left(\mathrm{mg} \mathrm{L}^{-1}\right)$ & $\mathrm{NO}_{3}^{-}\left(\mathrm{mg} \mathrm{L}^{-1}\right)$ & $\mathrm{SO}_{4}^{2-}\left(\mathrm{mg} \mathrm{L}^{-1}\right)$ & $\mathrm{EC}\left(\mu \mathrm{S} \mathrm{cm} \mathrm{cm}^{-1}\right)$ \\
\hline Event & \multicolumn{6}{|c|}{ Pre-event-water end member $\left(C_{\mathrm{P}}\right) \pm S E_{\mathrm{CP}}$} \\
\hline$\# 1$ & $-10.98 \pm 0.03$ & $-75.10 \pm 0.20$ & $162.84 \pm 1.84$ & $11.33 \pm 0.05$ & $20.22 \pm 1.27$ & $708.80 \pm 18.45$ \\
\hline$\# 2$ & $-10.61 \pm 0.07$ & $-73.36 \pm 0.29$ & $166.66 \pm 1.58$ & $13.85 \pm 0.12$ & $24.18 \pm 1.49$ & $718.40 \pm 14.39$ \\
\hline \#3 & $-10.71 \pm 0.03$ & $-73.57 \pm 0.18$ & $166.28 \pm 1.62$ & $12.84 \pm 0.10$ & $23.77 \pm 1.47$ & $711.20 \pm 14.28$ \\
\hline \#4 & $-10.77 \pm 0.05$ & $-73.74 \pm 0.32$ & $158.11 \pm 1.53$ & $11.18 \pm 0.08$ & $21.84 \pm 1.35$ & $667.00 \pm 13.88$ \\
\hline \#5 & $-10.78 \pm 0.03$ & $-74.24 \pm 0.20$ & $163.97 \pm 1.66$ & $12.94 \pm 0.18$ & $24.46 \pm 1.51$ & $700.40 \pm 14.01$ \\
\hline$\# 6$ & $-11.25 \pm 0.03$ & $-77.30 \pm 0.21$ & $137.90 \pm 1.86$ & $8.05 \pm 0.10$ & $14.23 \pm 0.92$ & $562.20 \pm 12.61$ \\
\hline$\# 7$ & $-10.93 \pm 0.03$ & $-75.52 \pm 0.18$ & $163.61 \pm 1.57$ & $11.52 \pm 0.12$ & $21.05 \pm 1.30$ & $694.40 \pm 13.93$ \\
\hline$\# 8$ & $1.06 \pm 0.04$ & $-76.20 \pm 0.21$ & $166.67 \pm 1.61$ & $11.66 \pm 0.10$ & $22.22 \pm 1.37$ & 14.09 \\
\hline Event & \multicolumn{6}{|c|}{ Event-water end member $\left(C_{\mathrm{E}}\right) \pm S E_{\mathrm{CE}}$ at maximum event-water fraction } \\
\hline$\# 1$ & $-12.95 \pm 0.25$ & $-93.36 \pm 4.23$ & $13.14 \pm 3.19$ & $0.38 \pm 0.22$ & $0.12 \pm 0.06$ & 24.97 \\
\hline$\# 2$ & $-5.62 \pm 0.30$ & $-55.71 \pm 2.57$ & .37 & 0.66 & $1.27 \pm 0.63$ & 0.69 \\
\hline \#3 & $-7.99 \pm 0.90$ & $-58.77 \pm 4.26$ & $16.31 \pm 0.89$ & $0.46 \pm 0.13$ & $0.07 \pm 0.04$ & $24.02 \pm 20.34$ \\
\hline \#4 & $-10.70 \pm 0.54$ & $-94.29 \pm$ & .69 & & \pm 0.04 & 20.06 \\
\hline$\# 5$ & $-5.92 \pm 0.09$ & $-46.96 \pm 1.32$ & 1.01 & 0.46 & $2.08 \pm 0.56$ & 20.09 \\
\hline$\# 6$ & $-11.87 \pm 0.40$ & $-86.22 \pm 2.21$ & $6.99 \pm 3.76$ & $0.31 \pm 0.16$ & $0.05 \pm 0.04$ & $10.58 \pm 20.92$ \\
\hline$\# 7$ & $-14.24 \pm 1.28$ & $-129.38 \pm 7.76$ & 2.41 & \pm 0.56 & $0.06 \pm 0.04$ & \pm 21.17 \\
\hline$\# 8$ & $-15.29 \pm 0.50$ & $-121.41 \pm 2.76$ & $12.07 \pm 2.62$ & $2.77 \pm 0.45$ & $0.13 \pm 0.14$ & $20.24 \pm 20.98$ \\
\hline Even & \multicolumn{6}{|c|}{ Streamwater end member $\left(C_{\mathrm{S}}\right) \pm S E_{\mathrm{CS}}$ at maximum event-water fraction } \\
\hline$\# 1$ & $-12.52 \pm 0.03$ & $-85.99 \pm 0.17$ & $78.00 \pm 0.78$ & $4.16 \pm 0.15$ & $7.12 \pm 0.46$ & 56.86 \\
\hline$\# 2$ & $-10.27 \pm 0.03$ & $-70.60 \pm 0.17$ & 0.66 & 0.03 & $5.12 \pm 0.34$ & $=5.28$ \\
\hline \#3 & $-9.57 \pm 0.03$ & $-67.26 \pm 0.17$ & 0.89 & $5.77 \pm 0.04$ & $10.03 \pm 0.67$ & $346.00 \pm 6.92$ \\
\hline \#4 & $-12.54 \pm 0.03$ & $-87.74 \pm 0.17$ & $=0.90$ & $5.39 \pm 0.04$ & $8.00 \pm 0.52$ & \pm 6.94 \\
\hline \#5 & $-10.20 \pm 0.03$ & $-68.66 \pm 0.17$ & 0.96 & 0.04 & $12.86 \pm 0.81$ & $425.00 \pm 8.50$ \\
\hline \#6 & $-11.58 \pm 0.03$ & $-80.06 \pm 0.17$ & $107.28 \pm 1.04$ & $5.48 \pm 0.04$ & $9.57 \pm 0.61$ & $336.00 \pm 6.72$ \\
\hline \#7 & $-15.48 \pm 0.03$ & $-109.94 \pm 0.17$ & $2 \pm 0.87$ & $4.58 \pm 0.04$ & $7.59 \pm 0.49$ & $366.00 \pm 7.32$ \\
\hline$\# 8$ & $-12.93 \pm 0.03$ & $-89.25 \pm 0.17$ & $130.86 \pm 1.25$ & $9.06 \pm 0.04$ & $14.83 \pm 0.93$ & $1577.00 \pm 26.76$ \\
\hline \multicolumn{7}{|c|}{ Maximum event-water fraction $F_{\mathrm{E}, \max } \pm S E_{\mathrm{FE}}(\%)$} \\
\hline Event & $\delta^{18} \mathrm{O}$ & $\delta^{2} \mathbf{H}$ & $\mathrm{Ca}^{2+}$ & $\mathrm{NO}_{3}^{-}$ & $\mathrm{SO}_{4}{ }^{2-}$ & EC \\
\hline$\# 1$ & $78.17 \pm 10.22$ & $59.65 \pm 13.87$ & \pm 1.42 & \pm 1.92 & $65.21 \pm 3.20$ & $55.58 \pm 2.66$ \\
\hline$\# 2$ & $6.72 \pm 1.49$ & $15.68 \pm 2.84$ & $67.70 \pm 1.20$ & $82.84 \pm 4.50$ & $83.17 \pm 2.95$ & $64.67 \pm 2.17$ \\
\hline$\# \mathbf{3}$ & $41.99 \pm 13.90$ & $42.61 \pm 12.33$ & $50.92 \pm 0.85$ & $57.08 \pm 0.77$ & $57.96 \pm 3.84$ & $53.15 \pm 2.11$ \\
\hline$\# 4$ & $-{ }^{\mathrm{a}}$ & $68.15 \pm 14.48$ & $45.45 \pm 0.98$ & $61.99 \pm 2.06$ & $63.53 \pm 3.28$ & $48.37 \pm 2.10$ \\
\hline$\# 5$ & $11.79 \pm 0.85$ & $20.44 \pm 1.30$ & $43.77 \pm 0.94$ & $40.35 \pm 2.31$ & $51.83 \pm 5.03$ & $39.92 \pm 2.09$ \\
\hline$\# 6$ & $52.06 \pm 34.19$ & $30.90 \pm 8.05$ & $23.39 \pm 1.51$ & $33.24 \pm 1.24$ & $32.92 \pm 6.13$ & $41.01 \pm 2.39$ \\
\hline$\# 7$ & $137.17 \pm 53.13$ & $63.92 \pm 9.22$ & $49.57 \pm 1.10$ & $69.19 \pm 3.93$ & $64.11 \pm 3.24$ & $48.56 \pm 2.15$ \\
\hline$\# 8$ & $44.35 \pm 5.29$ & $28.88 \pm 1.83$ & $23.16 \pm 1.20$ & $29.26 \pm 1.75$ & $33.45 \pm 5.89$ & $-94.65 \pm 6.37^{b}$ \\
\hline
\end{tabular}

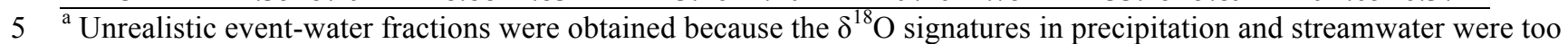
similar.

${ }^{\mathrm{b}}$ Wash-off of road salt resulted in unrealistic event-water fractions based on EC. 